\title{
Understanding tobacco smoke carcinogen NNK and lung tumorigenesis (Review)
}

\author{
GOHAR AKOPYAN and BENJAMIN BONAVIDA

\begin{abstract}
Department of Microbiology, Immunology and Molecular Genetics, David Geffen School of Medicine at UCLA, Jonsson Comprehensive Cancer Center, University of California, Los Angeles, CA 90095-1747, USA
\end{abstract}

Received May 17, 2006; Accepted June 19, 2006

\begin{abstract}
The deleterious effects of tumor-promoting tobacco carcinogen, nitrosamine 4-(methylnitrosamino)-1-(3-pyridyl)1-butanone (NNK, nicotine-derived nitrosamine ketone) have undoubtedly been stipulated. Though many tobacco agents play a part in the development of lung tumors, the potent effects of NNK remain unmatched. It is therefore critical to distinguish the variety of cofactors involved in NNK-mediated pathogenesis, and the unique pathways necessary for successful cellular biotransformation. Current reviews have consistently identified the strengths of NNK and prospective tumor capabilities. Others have delineated specific cellular factors mediating NNK and lung tumors, and have identified metabolic and signaling pathways largely responsible for NNK activation and tumorigenic initiation. Unique to this review is
\end{abstract}

Correspondence to: Professor Benjamin Bonavida, Department of Microbiology, Immunology and Molecular Genetics, David Geffen School of Medicine at UCLA, Jonsson Comprehensive Cancer Center, University of California, Los Angeles, CA 900951747, USA

E-mail: bbonavida@mednet.ucla.edu

Abbreviations: $\mathrm{AT}_{2}$, angiotensin II; Bcl2, B cell leukemia/ lymphoma 2; CC10, clara cell $10 \mathrm{kDa}$ protein; CIN, chromosome instability; c-Myc, cellular myelocytomatosis; CYP, cytochrome pigment 450, cytochrome pink 450; DAP, death-associated protein; EGFR, epidermal growth factor receptor; EPHX1, microsomal epoxide hydrolase; ERK1/2, extracellular signal-regulated protein kinase 1/2; FasL, Fas ligand; HDAC, histone deacytelase; IL-8, interleukin-8; IL-6, interleukin-6; ITC, isothiocyanate; KO, knockout; MAPK, mitogen-activated protein kinase; MCP-1, monocyte chemoattractant protein 1; MEK, MAPK/ERK kinase; MPO, myeloperoxidase; NFKB, nuclear factor kappa B; NHBE, normal human bronchial epithelial cells; NNAL, 4-(methylnitrosaminmo)-1-(3-pyridyl)-1-butanol; NNK, nitrosamine 4-(methylnitrosamino)-1-(3-pyridyl)-1-butanone, nicotine-derived nitrosamine ketone; NQO1, NAD(P)H quinone reductase 1; NSCLC, non-small cell lung cancer; $\mathrm{PGE}_{2}$, prostaglandin $\mathrm{E}_{2}$; PI3K/Akt, phosphoinositide 3-kinase, phosphatidylinositol 3-kinase; $\mathrm{PKC} \alpha$, protein kinase $C$ alpha; RAR- $\beta$, retinoic acid receptor beta; SCLC, small cell lung cancer; SAEC, small airway epithelial cells; SULT1A1, sulfotransferase $1 \mathrm{~A} 1$; TGF- $\mathrm{B}$, tumor growth factor beta; TNF, tumor necrosis factor; WT, wild-type

Key words: tobacco smoke, lung cancer, NNK that it summarizes the extensive network of cofactors and cellular mechanisms that promote NNK-specific lung tumorigenesis. As such, it displays a fuller, more comprehensive overview, bringing us one step closer to understanding the fatal consequences of NNK, thus, discovering new avenues that successfully break the cycle of NNK-mediated lung carcinogenesis.

\section{Contents}

1. Introduction

2. Activation of NNK-mediated lung tumorigenesis

3. Effects of NNK on cellular factors

4. Repression of NNK-mediated lung tumorigenesis

5. Concluding remarks

\section{Introduction}

Throughout the array of cancers worldwide, lung cancer is one of the most fatal. The close link between lung cancer and tobacco smoke is unequivocal (1-4). The first evidence of a direct correlation between inhaled tobacco smoke and lung cancer was demonstrated in human studies in Germany between 1930-1940; further studies were later confirmed in Great Britain and the US in the 1950s $(4,5)$. Despite the rise of anti-tobacco sentiment, cigarette smoke remains the most potent cause of lung cancer deaths. Approximately $90 \%$ of male and $75-80 \%$ of female lung cancer deaths are caused by smoking each year in the US (2). Numerous studies have confirmed these facts, but few have outlined the chief tobacco ingredients and associated biomolecular pathways leading to lung tumorigenesis. Although many factors in tobacco smoke contribute to lung cancer, the key ingredient, nitrosamine 4(methylnitro-samino)-1-(3-pyridyl)-1-butanone (NNK, nicotine-derived nitrosamine ketone) plays a major role in carcinogenesis.

The most carcinogenic molecules in tobacco smoke belong to two groups: i) polycyclic aromatic hydrocarbons and ii) nitrosamines $(4,6,7)$. While both have shown a significant relationship with lung tumorigenesis, nitrosamines have a remarkably larger effect. Specifically, studies on tobacco smoke have confirmed NNK as the most potent ingredient responsible for lung carcinogenesis $(2,8)$. It is also the only carcinogen that systemically induces tumors of the lung in 
rat, mouse, and hamsters - three of the most common rodent models (6).

\section{Activation of NNK-mediated lung tumorigenesis}

Structure. NNK is an aromatic compound with a molecular formula of $\mathrm{C}_{10} \mathrm{H}_{13} \mathrm{~N}_{3} \mathrm{O}_{2}(\mathrm{MW}=207.2316)$. This yellow crystalline solid is the most potent carcinogenic compound in the family of nitrosamines. The crystalline structure of NNK has been characterized (9) and the chemical structure is illustrated in Fig. 1.

\section{Factors associated with NNK activation}

Cofactors. Although NNK mediates rapid progression of lung cancer, cofactors responsible for the metabolism and activation of NNK are crucial for carrying out its functions. NNK initially presents itself in the body as a procarcinogen, an inert form that requires activation to exert its full effects $(7,10,11)$. Metabolic enzymes facilitating the activities of NNK are the main constituents through which NNK-specific metabolic pathways and subsequent NNK activation occur. Cytochrome pigment 450 (CYP450) enzymes belonging to the CYP multigene family, for example, catalyze the $\alpha$-hydroxylation of NNK in the oxidative metabolism pathway (7,10-12). Once NNK and NNK-type carcinogens become reactive in the process, they can easily bind to DNA, form DNA adducts, and initiate mutations responsible for tumor development $(7,10)$. A three-dimensional model of the metabolic activation of NNK via CYP enzyme, CYP2A13, is shown in Fig. 2 (13).

Phosphorylation and subsequent activation of nuclear factor kappa B (NFkB) may also be a consequence of NNKdependent processes (14), but the direct involvement of NNK in mediating such cofactors has been debated (15). More detailed explanations about the association between NNK and $\mathrm{NF} \kappa \mathrm{B}$ are provided in the signaling pathways subsection.

Metabolic genes. Activation of NNK may also be contingent upon metabolic genes. For example, molecular epidemiological studies of metabolic genes such as NAD $(\mathrm{P}) \mathrm{H}$ quinone reductase 1 (NQO1), myeloperoxidase (MPO) and microsomal epoxide hydrolase (EPHX1) have shown close involvement with metabolic activation and detoxification of aromatic amines $(7,11)$. Therefore, NNK is a likely candidate for genedependent activation.

\section{Pathways associated with NNK activation}

Metabolic pathways. NNK does not work in isolation; it requires cellular metabolism and activation to induce tumorigenesis. There are three primary pathways responsible for NNK activation: i) carbonyl reduction, ii) pyridine $\mathrm{N}$-oxydation and iii) $\alpha$-hydroxylation $(16,17)$ (Fig. 3). NNK undergoes cytochrome P450-catalyzed $\alpha$-hydroxylation by two pathways (either $\alpha$-methylhydroxylation or $\alpha$-methylenehydroxylation), both of which produce carcinogenic intermediates (16). Studies done on human bronchiolar and alveolar cell lines (BEAS2B and A549, respectively) treated with $500 \mu \mathrm{M}$ NNK, showed significant reduction in crucial immunoprotective cytokines such as interleukin-8 (IL-8) and interleukin-6 (IL-6); and mediators such as monocyte chemoattractant protein 1

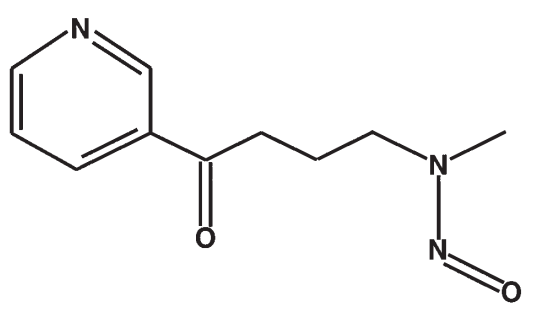

Figure 1. Chemical structure of NNK.

(MCP-1) (16). NNK induced these outcomes via both $\alpha$-hydroxylation pathways, independent of the presence or absence of cellular co-stimulation with $25 \mathrm{ng} / \mathrm{ml}$ of tumor necrosis factor (TNF). However, it upregulated prostaglandin $\mathrm{E}_{2}\left(\mathrm{PGE}_{2}\right)$ production, leading to further inhibition of tumor-cell apoptosis and increased cellular proliferation (16). The immunomodulatory effects of NNK are thus clearly visible; its potential to alter respiratory epithelial cells and lead to deficiencies of the immune response is one of many ways by which NNK works to strengthen and expedite lung tumor advancement.

NNK also undergoes reductive metabolism, and in the process produces its metabolized isoform, 4-(methylnitrosaminmo)-1-(3-pyridyl)-1-butanol (NNAL), a carcinogenic compound and metabolic product of NNK $\alpha$-hydroxylation (10). Further metabolic processing and detoxification of NNAL produces non-carcinogenic compounds known as NNAL-Glucs. Nonetheless, the glucuronidation of NNAL guided either by carbonyl reduction or pyridine N-oxydation (part of the reductive metabolic pathway) is an important mechanism of NNK detoxification, activation and consequent development of lung tumors (17). NNAL and NNAL-Glucs thus serve as biomarkers for NNK uptake via direct and second-hand tobacco smoke (6), an important method through which a direct, statistically-significant correlate is established between quantifiable measures of NNK metabolites and the intensity of smoking (10).

NNK's potent effects are not discriminative. Its ability to induce lung carcinogenesis is independent of the route of administration (17). Of note, concentrations of NNK have been described in tobacco substances such as snuff $(1-20 \mu \mathrm{g} / \mathrm{g})$, cigarette mainstream smoke (20-310 ng/cigarette), and tobacco-exposed areas such as indoor air $\left(\leq 26 \mathrm{ng} / \mathrm{m}^{3}\right)(9)$. Hence, it is to no surprise that both direct and second-hand tobacco smoke exposure lead to quantifiable measures of NNK, NNAL, and NNAL-Glucs in urine $(6,10,18)$. Urine testing and subsequent observation of tobacco smoke-related metabolites and carcinogens thus provides sufficient information about carcinogen dose (10), further confirming prospective lung cancer risks of smokers and non-smokers alike. Epidemiological studies on smokers of regular, light, and ultralight cigarettes have found no significant differences in lung cancer potential. Hecht and colleagues (1) did further analysis to confirm this by observing lung carcinogen uptake on smokers of the above three types. Upon analysis of urine samples of 175 recruited smokers of regular, light, and ultralight cigarettes, each of whom averaged 15-45 cigarettes per day, there was no significant difference in quantifiable measures of NNK-metabolites such as NNAL between the three types (an average of 2.1-3.2 pmol NNAL/mg of creatinine). Lung 

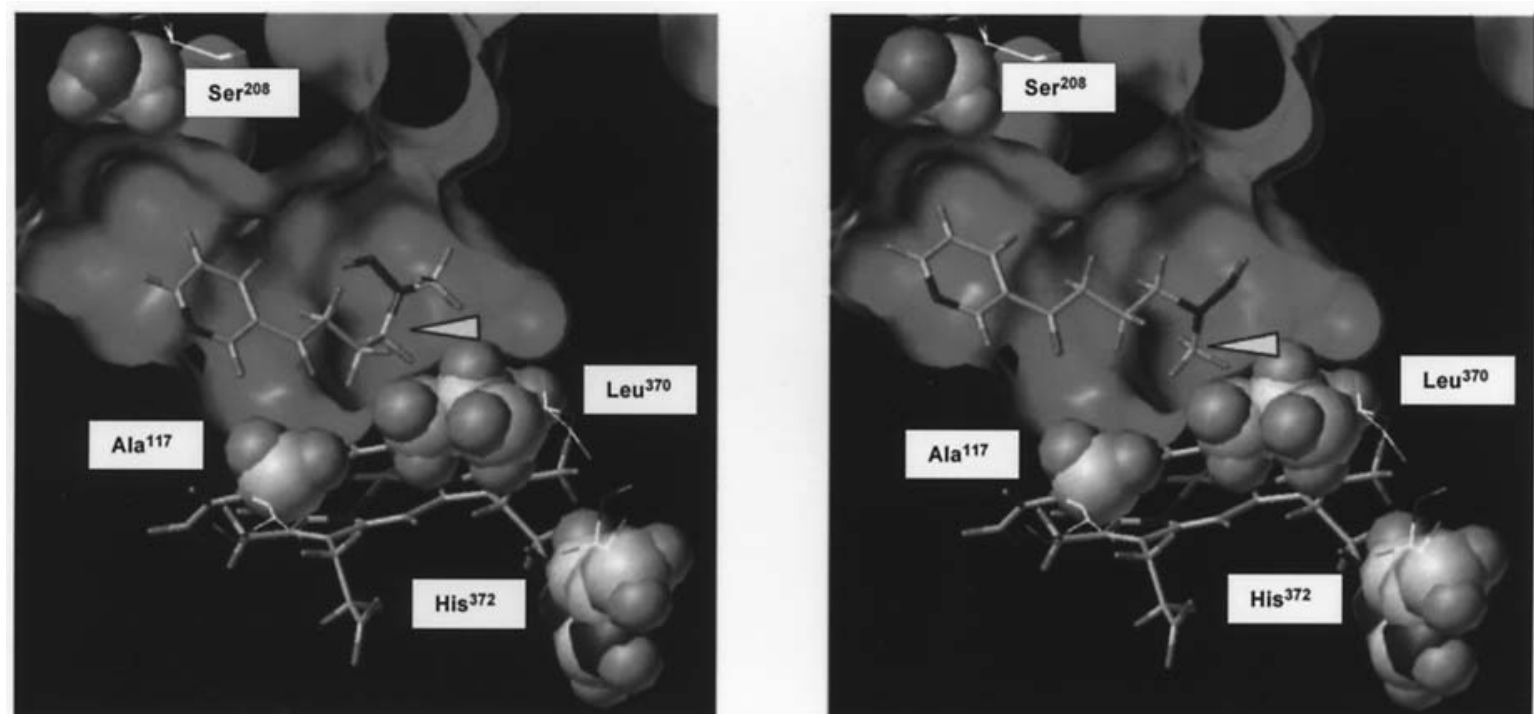

Figure 2. Crystal structure of NNK. CYP2A13 model with NNK docked into the active site. (Left) Methylene hydroxylation (for the formation of keto aldehyde); (Right) Methyl hydroxylation (for the formation of keto alcohol). The position of the methylene and the methyl carbons are indicated by arrowheads. Both binding models exhibit an extended conformation with the NNK nitroso group (head) toward the proximal pocket of the heme and the pyridine group (tail) pointing away from the heme. The long axis passing the head and tail forms a 30 degree angle with the heme plane. The distances from the heme iron to the reactive methylene carbon and methyl carbon are $4.3 \AA$ and $4.1 \AA$, respectively [Courtesy of He et al (13)].

$(7,11)$

NQO1, MPO, EPHX1

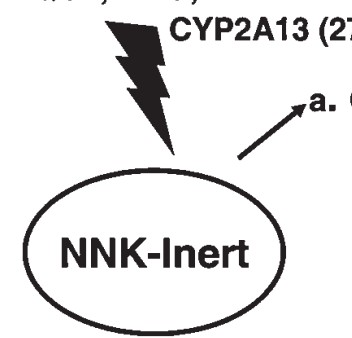

$(7,10,11)$
(7,10-12)

CYP450

Oxid. Metab.

$\longrightarrow \alpha$-Hydrox.

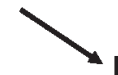

b. Red. Metab.
(16)

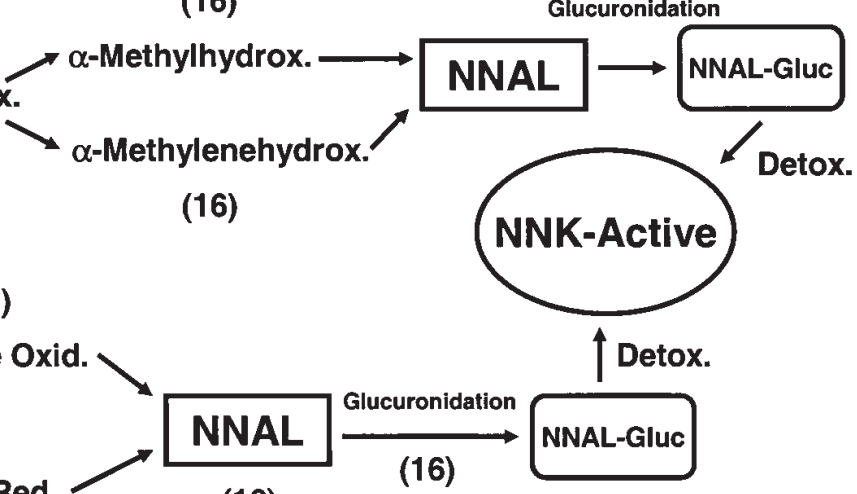

(10)

(16)

$(16,17)$

Figure 3. Activation of NNK via metabolic pathways. NNK initially presented as inactive form. Upon exposure to specific genes (NQ01, MPO, EPHX1) and enzymes (CYP2A13), NNK undergoes oxidative and reductive metabolism, leading to its metabolites, NNAL and NNAL-Glucs. Metabolite detoxification subsequently leads to the activation of NNK. References corresponding to mechanisms are cited in parentheses.

carcinogen uptake is therefore the same in smokers of regular, light, and ultralight cigarettes (1), signifying the strong potential of non-selective NNK-mediated lung tumorigenesis for smokers of all types.

Signaling pathways. The predisposition of NNK in driving lung tumor progression is highly correlated with several signaling pathways (Fig. 4). Clara cell $10 \mathrm{kDa}$ (CC10), for example, is an essential multifunctional protein secreted by airway epithelial cells. Potential damage to this protein may lead to increased proclivity for lung cancer development. For instance, Yang and colleagues (19) performed studies on wild-type (WT) and CC10-knockout (KO) mice exposed to NNK $(104 \mathrm{mg} / \mathrm{kg}$ of body weight, given 3 times every other month). CC10-KO mice had a much higher predisposition of airway epithelial hyperplasia and adenocarcinoma formation, mutations in the proto-oncogene $K$-ras, FasL overexpression, and mitogen-activated protein kinase/extracellular signalregulated kinase 1 (MAPK/ERK1) hyperphosphorylation relative to their CC10-WT counterparts (19), suggesting a crucial protective role of CC10 against NNK-induced lung hyperplasia and adenoma formation. Further studies demonstrate NNK-activated $\mu$ - and m-calpain kinases as necessary ingredients for NNK-induced lung metastasis via the ERK1/2 pathways (20).

Moreover, NNK has been shown to upregulate cellular myelocytomatosis (c-Myc) and B cell leukemia/lymphoma 2 $(\mathrm{Bcl} 2)$, two oncoproteins that assist one another in various 


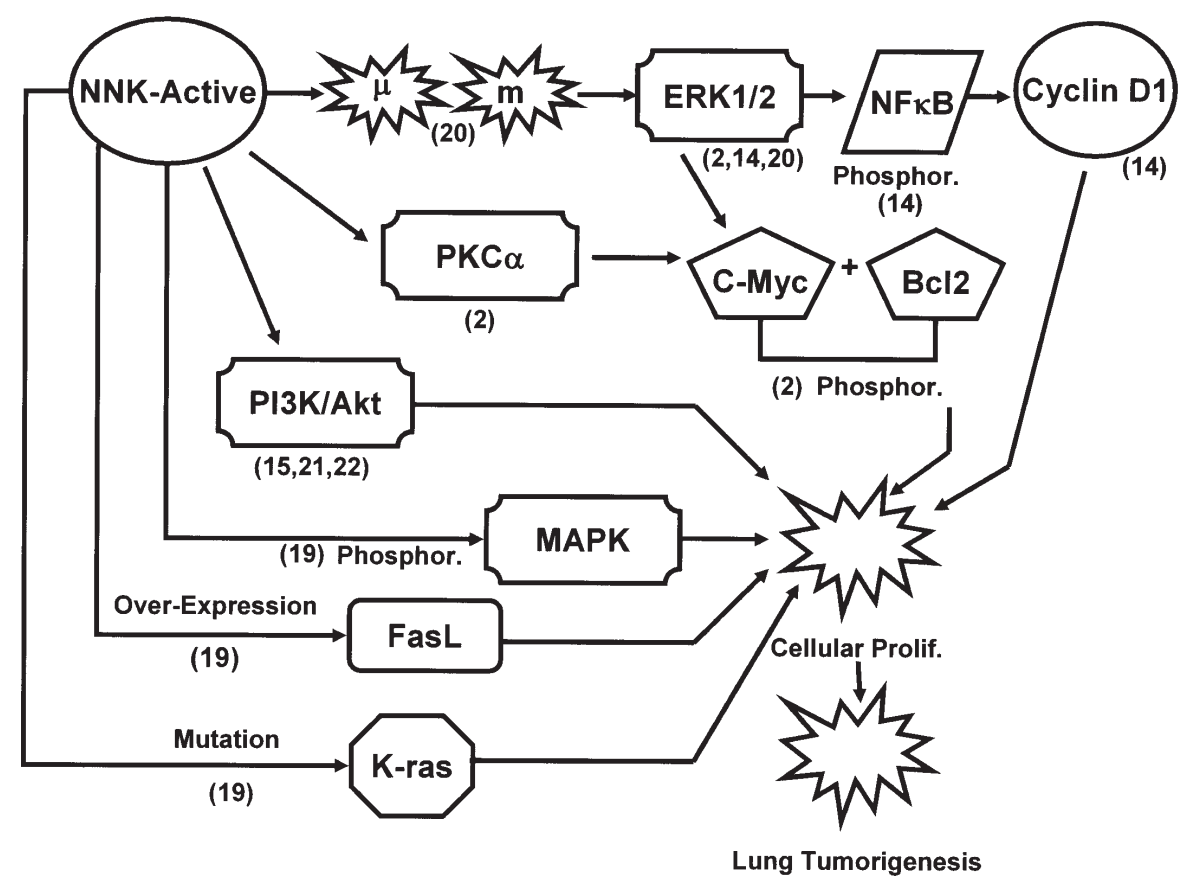

Figure 4. NNK-mediated activation of signaling pathways. Once activated, NNK initiates a cascade of signaling pathways (ERK1/2, PKC $\alpha$, PI3K/Akt, MAPK), resulting in uncontrolled cellular proliferation and tumorigenesis. References corresponding to mechanisms are cited in parentheses.

cellular processes including proliferation, transformation, apoptosis and tumorigenesis (2). By way of several cellular pathways, such as the protein kinase cascade, protein kinase C $\alpha /$ Raf-1/MAPK/ERK1/2 (PKC $\alpha /$ Raf-1/MEK 1/2), NNK successfully promotes cell survival via phosphorylation and subsequent functional cooperation of c-Myc and Bcl2 (2). Triggering such NNK-dependent events further leads to cellular migration, invasion, and uncontrolled proliferation.

ERK1/2 not only induces NNK-specific tumorigenic activities in a direct fashion, but also assists other cellular agents of which NNK directly stimulates. Studies done by Ho and colleagues (14), for example, demonstrated phosphorylation of $\mathrm{NF \kappa B}$ both on tobacco-specific small airway epithelial cells (SAEC) and normal human bronchial epithelial cells (NHBE) upon cellular exposure to varying concentrations of NNK (0.01-50 $\mu \mathrm{M})$. Activation of the transcription factor in turn upregulated cyclin D1, a G1 phase regulatory protein which contributes to stimulation of normal human bronchial cells. Subsequent treatment of cell lines with $10-20 \mu \mathrm{M}$ of various ERK1/2-specific inhibitors suppressed NNK-mediated NFKB phosphorylation (14), further confirming ERK1/2 kinase as a necessary intermediary between NFKB-dependent protein expression and NNK-dependent cellular proliferation.

Interestingly, other studies disproved the direct involvement of NFKB in assisting NNK-mediated cellular survival (15). To demonstrate this, nicotine- and NNK-exposed (10 $\mu \mathrm{M}$ and $100 \mathrm{nM}$, respectively) non-small cell lung cancer (NSCLC) and small cell lung cancer (SCLC) cells were treated with chemotherapeutic agents such as paclitaxel (100 nM) and etoposide $(100 \mu \mathrm{M})$. Upon measuring the effects of NNK, Tsurutani and colleagues (15) found that while nicotine mediated resistance to apoptosis on treated cells, NNK did not, even at high dosages. They also found that unlike NNK, nicotine worked via an NFKB-dependent pathway to induce cellular resistance to apoptosis and thus, cellular survival (15). Lack of NNK-mediated cellular survival on NSCLC and SCLC cell lines disproves NNK's direct involvement with NFkB-dependent cellular survival. Such analytical discrepancies in studies necessitate further in-depth observations to better understand NNK-mediated cellular resistance and the direct pathways utilized to accomplish it.

The phosphoinositide 3-kinase (PI3K/Akt) pathway is also a major contributor to NNK-induced cellular transformation and metastasis $(15,21)$. This process ensures the proliferation and survival of tumorigenic cells. West and colleagues (21) had previously demonstrated Akt-dependent protection of human lung epithelial cells of small and large airway origin against cellular stresses including DNA damage, serum withdrawal, and cell detachment. Analysis of the PI3/Akt pathway in human lung bronchial epithelial cell lines (BEAS2B) treated with $9.1 \mathrm{mg}$ of NNK in drinking water within an eight-week period led to increased cellular proliferation and survival as opposed to their untreated counterparts (21). PI3K/Akt pathway-dependent cellular survival was further confirmed by observing a significant decrease in PI3K/Akt phosphorylation of NNK-treated and untreated cell lines upon exposure to $2.5-10 \mu \mathrm{M}$ of PI3K/Akt inhibitors.

The Akt pathway is a molecular target of lung cancer; it is constitutively active in NSCLC cells in vivo and in vitro (22). Similar to the ERK1/2 pathway, the Akt pathway works with transcription factors such as NFkB to assist in carcinogenmediated activities. However, studies done on the Akt pathway have confirmed strengthening in cellular survival upon exposure to nicotine and NNK, but have established $\mathrm{NF \kappa B}$ as a necessary correlate only in nicotine-mediated cellular proliferation (15). 


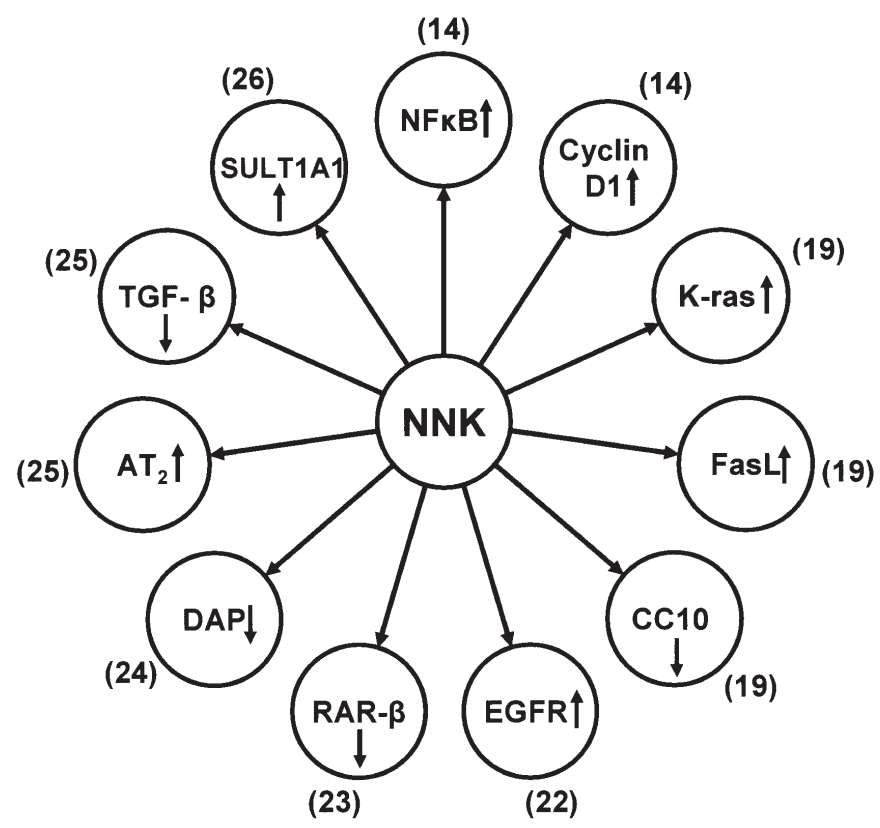

\section{$\uparrow$ = Activation or upregulation $\downarrow=$ Inactivation or downregulation}

Figure 5. Direct and indirect effects of NNK on various cellular factors. Key gene and protein factors associated with NNK functions are shown. Arrows represent the impact of NNK upon exposure to associated factors. Genes and proteins shown above undergo NNK-mediated polymorphisms and modifications, respectively. References corresponding to cellular factors are cited in parentheses.

The ERK1/2 and Akt pathways have both shown consequential changes in levels of protein expression as a result of carcinogen uptake by cells, but to say that a direct correlate always exists between these NNK-associated pathways and specific proteins is inconsistent. For example, cyclin D1 upregulation was a direct consequence of ERK1/2-dependent NNK-mediated NFKB phosphorylation (14). Akt-dependent cellular proliferation also induced changes in cyclin D1 expression (15), but the direct involvement of NNK in this process warrants thorough investigation. Significant changes in other cellular agents such as epidermal growth factor receptor (EGFR) have also been observed. Epithelial cell lines of small and large airways, for instance, have shown a higher level of EGFR phosphorylation upon exposure to carcinogenic compounds such as NNK (22). Although the Akt pathway has been observed during this process, a direct link between EGFR and Akt phosphorylation remains unexamined.

\section{Effects of NNK on cellular factors}

Genetic polymorphisms. Carcinogens like NNK are known for their notorious reputation as mutagens. NNK's ability to produce gene polymorphisms has been observed primarily in genes involving cellular function, growth, differentiation and proliferation. One of many NNK-dependent routes of cellular proliferation involves the downregulation of the retinoic acid receptor beta $(R A R-\beta)$ gene via methylation (23). Analysis of carcinomas induced by exposure to $100 \mathrm{mg} / \mathrm{kg}$ NNK for 14 weeks revealed significant point mutations of $R A R-\beta$, which further explains its inactivation during lung tumorigenesis. Other studies done on hyperplasias and adenocarcinomas, also a result of $100 \mathrm{mg} / \mathrm{kg}$ of NNK treatment, revealed abnormal promoter hypermethylation and resultant inactivation of deathassociated protein $(D A P)$-kinase, an important gene for cellular apoptosis (24). Additional studies done on target cells treated with NNK (104 mg/kg of body weight, given 3 times every other month) showed increased frequency of mutations in the proto-oncogene $K$-ras during early-phase alveolar adenomas (19). Kanehira and colleagues (25) also performed studies on mutations of the signaling gene, angiotensin II $\left(A T_{2}\right)$. Their results revealed a substantial decrease in susceptibility to NNKspecific lung carcinogenesis (induction dose of $100 \mathrm{mg} / \mathrm{kg}$ ) due to the gene's ability to modulate tumor growth factorbeta (TGF- $\beta$ ) production and subsequently increase cellular growth (25), implying a strong dependence of NNK on the functionality of this gene.

Genes heavily associated with NNK metabolic pathways are also prone to NNK-dependent mutations. The sulfotransferase 1A1 (SULT1A1) gene, for example, is an important factor of carcinogenic biotransformation (26). Polymorphisms in this gene substantially increased lung cancer susceptibility in smokers (26). Studies done by Su et al also demonstrated a direct involvement of NNK-mediated mutations on $C Y P 2 A 13$, a human cytochrome gene predominantly expressed in the respiratory tract (27). Because it has shown to be more active then other CYPs in NNK metabolism (28), it is safe to assume CYP2A13 as a crucial - if not the most important - CYP gene for NNK activation (10). Some studies have also looked at other CYP gene polymorphisms including CYPIAl and $C Y P 1 B 1$, both of which encode for CYP450 enzymes. Mutations on these genes may act as possible routes of aromatic amine-induced carcinogenesis (12). NNK can very well be a CYP gene-modification candidate as it is heavily dependent on CYP450-induced metabolic activation, but a direct link between NNK and CYP mutations is yet to be elucidated. The dense involvement of NNK on a variety of genes is clearly visible. NNK's potent ability to engage in gene silencing, modification, and functional disruption are all important elements that encourage early development of lung carcinogenesis. Fig. 5 summarizes influences on genes and proteins heavily involved with NNK-mediated functions.

Chromosomal aberrations. Exposure of NNK to DNA often leads to genomic instability. NNK's potency in manipulating gene functionality is not limited to an intact gene structure. In fact, many NNK-induced adenocarcinomas are related to the magnitude of chromosome instability (CIN) (29). Studies on NNK-exposed lung epithelial cells constitutively showed DNA damage upon exposure to $50 \mathrm{mg} \mathrm{NNK} / \mathrm{kg}$ body weight 3 times a week for eight weeks, suggesting the importance of NNK-mediated CIN initiation during lung tumorigenesis (29). Dense chromosomal aberrations and recruitment of genes with an altered DNA copy number under such circumstances are therefore inevitable.

NNK and alternative cancers. NNK's potent tumorigenic capabilities are not solely limited to the lung. Some studies have shown the dense involvement of NNK in tumors of the oral and nasopharyngeal regions. Arrendondo et al, for 
instance, found that NNK-mediated stimulation of nicotinic receptors expressed by the oral epithelial cell line, Het-1A, led to increased cellular proliferation and reduction in apoptosis (30). Others have found a significant correlation between urinary measures of NNAL/NNAL-Glucs (the two major metabolites of NNK) and oral leukoplakia (31).

Studies observing the effects of NNK exposure on the nasopharyngeal cavities have demonstrated a high proportion of NNK-activating nasal enzymes, which further justify NNK's tumorigenic impact in the nasal region. For example, Castonguay et al showed high activation levels of nasal enzymes mediating NNK's effects, an important mechanism of NNK-mediated neuroepitheliomas (32). Preston-Martin has also mentioned that a direct correlate between high levels of NNK and nasopharyngeal cancers exist (33), but more studies are needed to clearly elucidate NNK and the etiology of such cancers. Although NNK's potency in the progression and advancement of lung tumors has undoubtedly been stipulated, its ability to directly manipulate cellular functions and thus lead to cancers of the mouth or nasopharyngeal region require more elaborate and in-depth analyses than are currently available.

\section{Repression of NNK-mediated lung tumorigenesis}

Chemopreventive agents. NNK has numerous ways of directing both physical and genetic alterations that often lead to lung cancer. However, there are some protective agents against NNK-mediated functions. For example, Witsche (4) demonstrated the importance of beta-carotenes and $\mathrm{N}$-acetylcysteines against NNK-mediated lung cancer. In fact, diets rich in the above-mentioned ingredients resulted in a significant reduction of NNK concentration $(<20 \%)$ in mice previously exposed to filtered and non-filtered tobacco smoke (4).

Hecht et al demonstrated specific biomolecules that give cruciferous vegetables their anti-cancer properties. Cruciferous vegetables have a high dose of glucosynolates, plant defense compounds that hydrolyze into isothiocyanates (ITCs) and indole-3-carbinol (a product of hydrolyzed glucobrassicans), both of which act as chemopreventive agents against lung carcinogenesis (10). Upon analysis of vegetable consumption in smokers, Hecht and colleagues (10) found an indirect correlation between the intake of vegetables (with high levels of glucobrassicans) and urine traces of NNK metabolites (e.g. NNAL, NNAL-Glucs). Furthermore, NNK-exposed mice treated with indole-3-carbinol showed protection against lung tumor development by inducing hepatic $\alpha$ hydroxylation of NNK and therefore causing less NNK dispersion into the lungs (10). Conaway and colleagues looked at the chemotherapeutic potential of isothiocyanates more closely, and found a significant reduction in malignant lung adenocarcinomas on NNK-induced mice (34).

The above studies indicate the importance of anti-tumor agents in blocking or disrupting NNK-mediated lung cancer. Mechanisms such as distraction of NNK from respiratory dispersion via hepatic $\alpha$-hydroxylation, however, should be carefully monitored as they could minimize lung cancer susceptibility while maximizing the potential to develop liver disease. Thus, contraindications related to therapeutic agents may be unavoidable, further verifying the complex mechanisms involved for complete, risk-free inhibition of carcinogen-mediated tumorigenesis.

One of the most important ways to disrupt NNK behavior is by inhibiting its ability to methylate DNA. Histone deacytelase (HDAC) inhibitors, for example, have been used pharmacologically to aid in histone acetylation silencing mechanisms and act as demethylating agents (35). Tumor suppressor genes also play a crucial role, but are often inactivated by NNK-type carcinogens during lung tumorigenesis. Targeting NNK-specific oncogenes such as EGFR (35) and preventing hyperproliferation may become potential solutions.

Interruption of vital pathways. PI3K/Akt inhibitors such as deguelin $(36,37)$ work to paralyze NNK by blocking one of the most fundamental pathways liable for NNK activation. Studies with tamoxifen-treated transgenic mice expressing the Akt pathway revealed a statistically significant amount of NNK repression and NNK-mediated lung tumor multiplicities in vivo when exposed to deguelin (37). Further studies by Hecht (6) showed deguelin-mediated inhibition of the PI3/Akt pathway and apoptotic upregulation in premalignant and malignant human bronchial epithelial cells. Deguelin has also been implied as a possible candidate for inhibiting i) the CYP cytochrome family, ii) the induction of carcinogendetoxifying enzymes, or iii) the upregulation of DNA repair (36). These processes, however, are currently not well understood. Nevertheless, P13/Akt pathways are indispensible to many NNK-mediated tumors. As such, inhibitors such as deguelin may possibly be the most successful therapeutic mechanisms against NNK.

\section{Concluding remarks}

The relevance of NNK in triggering uncontrolled cellular proliferation and subsequent lung tumorigenesis has been consistently described and confirmed in numerous studies. Several mechanisms against cellular disruption via NNK and NNK-associated pathways have been proposed, including: i) blockage of metabolic and signaling pathways, ii) interruption and/or silencing of genes encoding enzymes coupled with NNK, iii) direct manipulation of enzymes (specifically from the CYP protein family) responsible for activation and initiation of NNK-mediated processes, and iv) NNK-specific inhibitors in food products. However, these primary prevention techniques have not yet been fully explored. Additional suggestions for combating NNK and its tumorigenic effects include i) generating tobacco whereby NNK is rendered inactive by structural and functional alteration; ii) adding chemotherapeutic ingredients to tobacco (e.g. deguelin, glucobrassicans) for immediate obstruction of NNK functions prior to entrance into the lungs; or iii) chemical modification of tobacco such that it lacks NNK all together.

Although the above-mentioned methods provide opening possibilities for inactivation of major carcinogens such as NNK, shortcomings of these models, nevertheless, exist. NNK is the dominant carcinogenic agent in tobacco smoke and thus the key player in lung cancer associated with smoking. However, the effects of tobacco factors such as nicotine and polycyclic aromatic compounds, must also be taken into 
consideration. While additional approaches for disrupting the biochemical properties and activities of other carcinogenic ingredients have been defined, fighting the effects of NNK nonetheless remains the most crucial step against lung tumor development. Currently, smokers who later develop lung cancer undergo secondary prevention treatments that focus on chemotherapy, radiotherapy, and pulmonary surgery. However, such methods fall short of perfect. In spite of progressive treatments, lung cancer still remains one of the most deadly cancers with poor prognosis and high mortality. Therefore, it is necessary to delineate the most potent biomarkers of lung tumorigenesis, and to develop efficient methods for their eradication.

Despite evident limitations, the above-mentioned models for primary prevention provide building blocks to fight against NNK, the major toxin in tobacco smoke. Interruption of key mechanisms linked to the optimization of NNK-dependent functions can successfully reduce the developing rate of lung cancer. Designing prospective therapeutics that emphasize lung tumor chemoprevention thus holds a promising future against the threats of NNK.

\section{Acknowledgements}

We acknowledge the assistance of Maggie Yang in the preparation of the manuscript.

\section{References}

1. Hecht SS, Murphy SE, Carmella SG, Li S, Jensen J, Le C, Joseph AM and Hatsukami DK: Similar uptake of lung carcinogens by smokers of regular, light, and ultralight cigarettes. Cancer Epidemiol Biomarkers Prev 14: 693-698, 2005.

2. Jin Z, Gao F, Flagg T and Deng X: Tobacco-specific nitrosamine 4-(methylnitrosamino)-1-(3-pyridyl)-1-butanone promotes functional cooperation of $\mathrm{Bcl} 2$ and $\mathrm{c}-\mathrm{Myc}$ through phosphorylation in regulating cell survival and proliferation. J Biol Chem 279: 40209-40219, 2004.

3. Panani AD and Roussos C: Cytogenetic and molecular aspects of lung cancer. Cancer Lett (Epub ahead of print).

4. Witschi H: Carcinogenic activity of cigarette smoke gas phase and its modulation by beta-carotene and $\mathrm{N}$-acetylcysteine. Toxicol Sci 84: 81-87, 2005.

5. Doll R: Uncovering the effects of smoking: historical perspective. Stat Methods Med Res 7: 87-117, 1998.

6. Hecht SS: Carcinogen derived biomarkers: applications in studies of human exposure to secondhand tobacco smoke. Tob Control (Suppl 1): i48-i56, 2004.

7. Kiyohara C, Yoshimasu K, Takayama K and Nakanishi Y: EPHX1 polymorphisms and the risk of lung cancer: a HuGE review. Epidemiology 17: 89-99, 2006.

8. Maser E: Significance of reductases in the detoxification of the tobacco-specific carcinogen NNK. Trends Pharmacol Sci 25: 235-237, 2004.

9. Katz AK, Carrell HL, Afshar CE, Glusker JP, Desai D and Amin S: 4-(methyl-nitrosamino)-1-(3-pyridyl)-1-butanone, a nicotine-derived carcinogenic nitrosaminoketone (NNK): threedimensional structure. Struct Chem 10: 439-443, 1999.

10. Hecht SS, Carmella SG, Kenney PM, Low SH, Arakawa K and $\mathrm{Yu}$ MC: Effects of cruciferous vegetable consumption on urinary metabolites of the tobacco-specific lung carcinogen 4(methylnitrosamino)-1-(3-pyridyl)-1-butanone in singapore chinese. Cancer Epidemiol Biomarkers Prev 13: 997-1004, 2004.

11. Kiyohara C, Yoshimasu K, Takayama K and Nakanishi Y: NQO1, MPO, and the risk of lung cancer: a HuGE review. Genet Med 7: 463-478, 2005.

12. Wenzlaff AS, Cote ML, Bock CH, Land SJ, Santer SK, Schwartz DR and Schwartz AG: CYP1A1 and CYP1B1 polymorphisms and risk of lung cancer among never smokers: a population-based study. Carcinogenesis 26: 2207-2212, 2005.
13. He XY, Shen J, Ding X, Lu AY and Hong JY: Identification of critical amino acid residues of human CYP2A13 for the metabolic activation of 4-(methylnitrosamino)-1-(3-pyridyl)-1butanone, a tobacco-specific carcinogen. Drug Metab Dispos 32: 1516-1521, 2004

14. Ho YS, Chen CH, Wang YJ, Pestell RG, Albanese C, Chen RJ, Chang MC, Jeng JH, Lin SY, Liang YC, Tseng H, Lee WS, Lin JK, Chu JS, Chen LC, Lee CH, Tso WL, Lai YC and Wu CH: Tobacco-specific carcinogen 4-(methylnitrosamino)-1-(3pyridyl)-1-butanone (NNK) induces cell proliferation in normal human bronchial epithelial cells through NFkappaB activation and cyclin D1 up-regulation. Toxicol Appl Pharmacol 205: 133-148, 2005.

15. Tsurutani J, Castillo SS, Brognard J, Granville CA, Zhang C, Gills JJ, Sayyah J and Dennis PA: Tobacco components stimulate Akt-dependent proliferation and NFkappaB-dependent survival in lung cancer cells. Carcinogenesis 26: 1182-1195, 2005.

16. Proulx LI, Gaudreault M, Turmel V, Augusto LA, Castonguay A and Bissonnette EY: 4-(Methylnitrosamino)-1-(3-pyridyl)-1butanone, a component of tobacco smoke, modulates mediator release from human bronchial and alveolar epithelial cells. Clin Exp Immunol 140: 46-53, 2005.

17. Wiener D, Doerge DR, Fang JL, Upadhyaya P and Lazarus P: Characterization of N-glucuronidation of the lung carcinogen 4(methylnitrosamino)-1-(3-pyridyl)-1-butanol (NNAL) in human liver: importance of UDP-glucuronosyltransferase 1A4. Drug Metab Dispos 32: 72-79, 2004

18. Tulunay OE, Hecht SS, Carmella SG, Zhang Y, Lemmonds C, Murphy S and Hatsukami DK: Urinary metabolites of a tobaccospecific lung carcinogen in nonsmoking hospitality workers. Cancer Epidemiol Biomarkers Prev 14: 1283-1286, 2005.

19. Yang Y, Zhang Z, Mukherjee AB and Linnoila RI: Increased susceptibility of mice lacking Clara cell $10-\mathrm{kDa}$ protein to lung tumorigenesis by 4-(methylnitrosamino)-1-(3-pyridyl)-1butanone, a potent carcinogen in cigarette smoke. J Biol Chem 279: 29336-29340, 2004.

20. Xu L and Deng X: Tobacco-specific nitrosamine 4-(methylnitrosamino)-1-(3-pyridyl)-1-butanone induces phosphorylation of mu- and m-calpain in association with increased secretion, cell migration, and invasion. J Biol Chem 279: 53683-53690, 2004.

21. West KA, Linnoila IR, Belinsky SA, Harris CC and Dennis PA: Tobacco carcinogen-induced cellular transformation increases activation of the phosphatidylinositol 3'-kinase/Akt pathway in vitro and in vivo. Cancer Res 64: 446-451, 2004.

22. West KA, Linnoila IR, Brognard J, Belinsky S, Harris C and Dennis PA: Tobacco carcinogen-induced cellular transformation increases Akt activation in vitro and in vivo. Chest 125: 101S-102S, 2004.

23. Vuillemenot BR, Pulling LC, Palmisano WA, Hutt JA and Belinsky SA: Carcinogen exposure differentially modulates RAR-beta promoter hypermethylation, an early and frequent event in mouse lung carcinogenesis. Carcinogenesis 25: 623-629, 2004.

24. Pulling LC, Vuillemenot BR, Hutt JA, Devereux TR and Belinsky SA: Aberrant promoter hypermethylation of the deathassociated protein kinase gene is early and frequent in murine lung tumors induced by cigarette smoke and tobacco carcinogens. Cancer Res 64: 3844-3848, 2004.

25. Kanehira T, Tani T, Takagi T, Nakano Y, Howard EF and Tamura M: Angiotensin II type 2 receptor gene deficiency attenuates susceptibility to tobacco-specific nitrosamineinduced lung tumorigenesis: involvement of transforming growth factor-beta-dependent cell growth attenuation. Cancer Res 65: 7660-7665, 2005

26. Liang G, Miao X, Zhou Y, Tan W and Lin D: A functional polymorphism in the SULT1A1 gene (G638A) is associated with risk of lung cancer in relation to tobacco smoking. Carcinogenesis 25: 773-778, 2004.

27. Su T, Bao Z, Zhang QY, Smith TJ, Hong JY and Ding X: Human cytochrome P450 CYP2A13: predominant expression in the respiratory tract and its high efficiency metabolic activation of a tobacco-specific carcinogen, 4-(methylnitrosamino)-1-(3pyridyl)-1-butanone. Cancer Res 60: 5074-5079, 2000.

28. Cauffiez C, Lo-Guidice JM, Quaranta S, Allorge D, Chevalier D, Cenee S, Hamdan R, Lhermitte M, Lafitte JJ, Libersa C, Colombel JF, Stucker I and Broly F: Genetic polymorphism of the human cytochrome CYP2A13 in a French population: implication in lung cancer susceptibility. Biochem Biophys Res Commun 317: 662-669, 2004. 
29. Herzog CR, Desai D and Amin S: Array CGH analysis reveals chromosomal aberrations in mouse lung adenocarcinomas induced by the human lung carcinogen 4-(methylnitrosamino)1-(3-pyridyl)-1-butanone. Biochem Biophys Res Commun 341: 856-863, 2006.

30. Arredondo J, Chernyavsky AI and Grando SA: Nicotinic receptors mediate tumorigenic action of tobacco-derived nitrosamines on immortalized oral epithelial cells. Cancer Biol Ther 5: 511-517, 2006.

31. Kresty LA, Carmella SG, Borukhova A, Akerkar SA, Gopalakrishnan R, Harris RE, Stoner GD and Hecht SS: Metabolites of a tobacco-specific nitrosamine, 4-(methylnitrosamino)-1-(3-pyridyl)-1-butanone (NNK), in the urine of smokeless tobacco users: relationship between urinary biomarkers and oral leukoplakia. Cancer Epidemiol Biomarkers Prev 5: 521-525, 1996.

32. Castonguay A, Tjalve $\mathrm{H}$ and Hecht SS: Tissue distribution of the tobacco-specific carcinogen 4-(Methylnitrosamino)-1-(3pyridyl)-1-butanone and its metabolites in F344 rats. Cancer Res 43: 630-638, 1983.
33. Preston-Martin S: N-nitroso compounds as a cause of human cancer. IARC Sci Publ 84: 477-484, 1987.

34. Conaway CC, Wang CX, Pittman B, Yang YM, Schwartz JE, Tian D, McIntee EJ, Hecht SS and Chung FL: Phenethyl isothiocyanate and sulforaphane and their N-acetylcysteine conjugates inhibit malignant progression of lung adenomas induced by tobacco carcinogens in $\mathrm{A} / \mathrm{J}$ mice. Cancer Res 65 : 8548-8557, 2005.

35. Digel W and Lubbert M: DNA methylation disturbances as novel therapeutic target in lung cancer: preclinical and clinical results. Crit Rev Oncol Hematol 55: 1-11, 2005.

36. Hecht SS: Deguelin as a chemopreventive agent in mouse lung tumorigenesis induced by tobacco smoke carcinogens. J Natl Cancer Inst 97: 1634-1635, 2005.

37. Lee HY, Oh SH, Woo JK, Kim WY, Van Pelt CS, Price RE, Cody D, Tran H, Pezzuto JM, Moriarty RM and Hong WK: Chemopreventive effects of deguelin, a novel Akt inhibitor, on tobacco-induced lung tumorigenesis. J Natl Cancer Inst 97: 1695-1699, 2005. 